\title{
TETRAPLOIDÍA EN CERATOPHRYS (ANURA, LEPTODACTYLIDAE), ANÁLISIS DEL REGISTRO FÓSIL
}

\author{
Isabel T. Mercadal de Barrio ${ }^{1}$ \\ Alfonso Barrio ${ }^{1}$
}

\begin{abstract}
TETRAPLOIDY IN CERATOPHRYS (ANURA, LEPTODACTYLIDAE), ANALYSIS OF THE FOSSIL RECORD. It has been confirmed the existence of the tetraploid counterpart of the genus Ceratophrys Wied, 1824 (extint at the present time) in Argentina and Brazil since the Pliocene, and the apparition of the octaploidy in the Upper Pleistocene - Holocene. Fossil material of the diploid form, distinctive of the Chacoan District, has been reported from the horizons of the Montehermosense Formation in south-east of the province of Buenos Aires, Argentina. The coexistence of diploid-tetraploid and tetraploid-octaploid forms in the same province was documented.
\end{abstract}

KEYWORDS. Amphibia, Ceratophrys, fossil, osteocyte lacunae, tetraploidy.

\section{INTRODUCCIÓN}

La poliploidía constituye un mecanismo evolutivo, que ocurre tanto en algunos grupos vegetales como animales. Uno de los papeles evolutivos de la poliploidía es proveer de loci para las radiaciones adaptativas (MORESCALCHI, 1977) y explotar diversos recursos, de los que sus "padres" diploides no pudieron aprovechar. Los genes duplicados por poliploidía pueden retener su función original o una similar, lo cual se traduciría en una diversificación de las proteínas funcionales y regulatorias, o algunas copias podrían ser silenciadas por medios epigenéticos o mutacionales (WENDEL, 2000). En plantas la poliploidía les conferiría, además de las ventajas adaptativas al medio donde se desarrollan mayor vigor, tamaño y otras características deseables para el hombre en aquellas plantas de valor comercial (SteBbins, 1966). La presencia del fenómeno de poliploidía en vertebrados ha resultado ser particularmente enigmático ya que no se ha podido esclarecer su significado, obviando por supuesto su potencial valor evolutivo. En los anfibios anuros, se han barajado algunas hipótesis sobre su posible establecimiento (Bogart \& Wasserman, 1972; Morescalchi, 1977; Bogart, 1980; Reumer \& ThiéBaud, 1987) y los procesos de silenciamiento de sus genes, pero nunca fueron planteadas las causas o mecanismos de dicha instauración. Se los ha estudiado desde el punto de vista citogenético, bioacústico, ecológico, fisiológico y molecular, pero hoy en día son pocos

1. Museo Argentino de Ciencias Naturales "Bernardino Rivadavia", Av. Angel Gallardo 470, C 1405 DJR Buenos Aires, Argentina. (batracios@escape.com.ar) 
los autores que tratan sobre el tema, especialmente a lo que concierne al grupo animal.

Ceratophrys Wied, 1824 es uno de los diez géneros de anuros vivientes donde se han detectado casos de poliploidía y particularmente en este género algunas de sus especies poseen un nivel de ploidía extrema (diploide-octoploide). Otro género con especies con ploidía de diverso orden es el africano Xenopus Wagler, 1827 en el cual no sólo se han realizado estudios cromosómicos, citometría somática y espermática, sino también del tamaño de las lagunas óseas en organismos vivos. Tal como el género anterior, se ha registrado el hallazgo de fósiles, en este caso, no sólo en Sudamérica sino también en África (Estes, 1975).

En especies naturales de anfibios de un mismo género existe cierta uniformidad en cuanto al volumen nuclear y en aquellos géneros con especies poliploides existe además cierta correspondencia entre el nivel de ploidía y el volumen nuclear (contenido nuclear de ADN), tal lo comprobado en eritrocitos de Ceratophrys tanto vivos como preservados (Mercadal, 1981) y en material vivo (FrankHauser, 1945; MaCgregor \& Uzzel, 1964; Uzzel et al.,1975; Günther, 1977; George \& Lennartz, 1980). Reumer \&Thiébaud (1987) pretendieron establecer si existía alguna relación entre el tamaño de la laguna ósea y el nivel de ploidía en Xenopus. Utilizaron ejemplares vivientes, pero con vistas a que si la relación es lo suficientemente cuantificable, ésta podría permitir la determinación del nivel de ploidía en especímenes fósiles, teniendo en tal caso, como fuera puntualizado por VIALLI (1971) "importantes inferencias en el estudio de la evolución de vertebrados, cuando es aplicado a taxa fósiles".

Esta investigación del nivel de ploidía a través de la medición de las lagunas óseas, único "rastro fósil" reflejo de dicho estado, tiene por objeto conocer la "historia" del fenómeno de la poliploidía, en el anuro sudamericano Ceratophrys.

\section{MATERIAL Y MÉTODOS}

Se utilizó la técnica de Reumer \&Thiébaud (1987) para especies vivientes del género Xenopus, con modificaciones. Se extrajeron pequeños fragmentos óseos únicamente de la parte más fina del cráneo (máximo aproximado $2 \mathrm{~mm}^{2}$ ), tratando de elegirlos de modo tal de no que no fuese significativa para posteriores estudios taxonómicos. Se obtuvieron tanto trozos delgados como gruesos, debiendo ser procesados. Para ello fue utilizada una piedra de grano muy fino que se usa normalmente para afilar cuchillas de micrótomo. Se procedió a su abrasión presionando el trozo de hueso con la yema de un dedo hasta obtener un espesor de 0,02 mm. Se coloreó empleando la técnica de Reumer \&ThiéBAud (1987), respetando los tiempos de la técnica para el material procedente de yacimientos más recientes. Para ejemplares de mayor antigüedad (Montehermosense) que presentaban mayor mineralización, se los coloreaba con tionina durante 20 a 30 min y ácido pícrico 10 min o dependiendo de las características del fragmento, se optaba por desgastarlo y se lo deshidrataba directamente para su montaje. Las mediciones de las lagunas óseas fueron hechas utilizando microscopio binocular con micrómetro ocular. Se tomó como criterio medir todas aquellas lagunas óseas "buenas" que se encontraran en el preparado, variando por lo tanto el número de mediciones en todos los casos. Son consideradas como "buenas" a aquellas lagunas óseas cuyo borde se mantuvo nítido, en toda su extensión, al ajustar éste con el micrómetro. Es decir que las lagunas óseas se encuentren paralelas a la superficie de abrasión, evitando así errores por distorciones en la medición (largo y ancho) debido a la ubicación de la laguna en distintos planos del ojo del observador. El volumen de la laguna ósea fue calculado por la fórmula de un elipsoide $4 / 3 \pi(1 / 2 \mathrm{~L})(1 / 2 \mathrm{~A})^{2}$, siendo L el diámetro mayor y A, el menor (Mercadal, 1981).

El material examinado y analizado se encuentra depositado en los museos: The Natural History Museum (BMNH), Londres; Museo de La Plata (MLP), Buenos Aires y Museo Argentino de Ciencias Naturales "Bernardino Rivadavia" (MACN), Buenos Aires. Ejemplares fósiles: Ceratophrys cornuta Linnaeus, 1758: BRASIL, Minas Gerais: Lagoa Santa, Piso Pleistoceno (BMNH 18895). Ceratophrys 
ornata Bell, 1943: ARgentinA, Buenos Aires: G. Chávez, Los Tres Reyes, Piso Pleistoceno tardío-Holoceno (MLP 86.VIII.1.4), (MLP 86.VIII.1.5/6). Ceratophrys sp.: ARGENTINA, Buenos Aires: Olavarría, Tapalqué, Piso Pleistoceno (MLP 86.III.25.150), (MLP 86.III.25.151); Gral. La Madrid, Fortín Necochea, Piso Holoceno temprano-medio (MLP 96.V.18.12); Mar Chiquita, Playa Dorada, Piso Ensenadense (MACN 19744); Gral. Pueyrredón, Mar del Plata, Piso Ensenadense (MLP 88.V.20.1); Chapadmalal, Piso Chapadmalalense (MACN 18074); Arroyo Lobería, Piso Marplatense, Barrancalobense (MACN 17585); Pta. Lobería, Piso Chapadmalalense (MACN 17936); Punta Varohué, Piso Chapadmalalense (MLP 34.V.10.8); Miramar, Barranca Parodi, Piso Chapdmalalense (MLP 88.VII.20.1), (MLP 88.VII.20.2); Miramar, Piso Holoceno superior (MLP 52.IX.27.11); sin citas de localidad y piso (MACN 19731); Quequén Salado, Piso Chapadmalalense inferior (MLP 94.II.1.171); Monte Hermoso, Piso Montehermosense (MLP 48.XII.16.195), (MACN 14322); Monte Hermoso, Piso no citado (presumiblemente Pleistoceno) (MLP 136), (MLP 247). Ceratophrys prisca Ameghino, 1899: ARGENTINA, Buenos Aires: Monte Hermoso, Piso Montehermosense (MACN 14324), (MACN 14317, plesiotipo). Ceratophrys prisca var. subcornuta Rovereto, 1914: ARGENTINA, Buenos Aires: Monte Hermoso, Piso Montehermosense (MACN 14319), (MACN 14323), (MACN 14325). Ceratophrys prisca var. gigantea Rovereto, 1914: ARGENTINA, Buenos Aires: Monte Hermoso, Piso Montehermosense (MACN 14318).

Fueron tomados como referencia del tamaño de las lagunas óseas cráneos de ejemplares diploides y octoploides actuales: Ceratophrys cranwelli Barrio, 1980: ARGENTINA, Chaco: Castelli (MACN, Lab. 80, A), (MACN, Lab. 80, B). Ceratophrys ornata Bell, 1843: ARGENTINA, Buenos Aires: Ing. Maschwitz (MACN, Lab. 80, A), (MACN, Lab. 80, B).

Material importante, tanto desde el punto de vista de la ubicación geográfica como de su antigüedad, no pudo ser examinado: Ceratophrys sp. del Plioceno (Montehermosense) de Catamarca, Argentina (Marshall \& Patterson, 1981, depositado en el Field Museum de Chicago (FMNH P 14402, parte de cráneo), extraviado (J. Bolt, comunic. epistolar). Ceratophrys sp. del Pleistoceno de Bolivia (Ñuapua y Tarija) estudiados por Vergnaud-Grazzini (1968): el Dr. Jean-Claude Rage (Laboratoire de Paleontologie, Museum National d'Histoire Naturelle) confirma que deben ser localizados. Ceratophrys ensenadensis Rusconi, 1932 de los alrededores de la ciudad de Buenos Aires (MACN), no fue posible localizarlo. El holotipo de Wawelia gerholdi Casamiquela, 1963 (MLP), no fue posible realizar ninguna observación (a través de un videomicroscopio de los rastros de estructuras óseas dejadas en la impronta) ya que le fuera aplicado un molde de látex que la inutilizó totalmente para los fines; Ceratophrys sp. (BMNH) procedente del Pleistoceno de Brasil (GüNTHER, 1859), los índices fueron calculados en base a fotografías de cráneos de dos ejemplares, sin embargo el material óseo enviado correspondía a un tercer ejemplar, no pudiendo así establecer ninguna correspondencia con los índices morfométricos.

En algunos ejemplares fue posible la medición de caracteres morfométricos craneanos a fin de establecer índices que posibilitarían compararlos con los obtenidos en las especies de Ceratophrys actuales. Como patrón de referencia, fueron considerados los índices obtenidos para $C$. ornata y $C$. cranwelli por BARRIO (1980), a fin de poder establecer alguna relación entre el parentesco y el nivel de ploidía (índices 3, 4 y 5). A éstos los denominamos índices A juntamente con otros, obtenidos de mediciones no consideradas en la metodología clásica (escotadura postorbitaria/distancia entre las puntas de los escamosales, distancia entre punta del escamosal y cuadrado/altura del neurocráneo, ancho del cráneo/ancho foramen magnum, ancho del cráneo/ancho de la órbita y distancia interorbital/ ancho de la órbita). Algunos de ellos fueron a su vez combinados para acentuar dichas disimilitudes (tab. II, índices B). De los 31 ejemplares estudiados sólo en 13 pudieron ser calculados todos los índices por encontrarse sus cráneos bastante completos. Teniendo en cuenta que las diferencias de los índices craneales de C. ornata y C. cranwelli son significativas (BARRIO, 1980), fueron consideradas como referencia para establecer similitudes con los fósiles estudiados.

\section{RESULTADOS}

El cálculo del nivel de ploidía a través de las mediciones de las lagunas óseas de fósiles de Ceratophrys indicaría la existencia de una forma con nivel de ploidía intermedio entre los clásicamente conocidos diploide y octoploide, es decir tetraploides (tab. I). 
Tabla I. Ejemplares estudiados actuales y fósiles del género Ceratophrys, su ubicación geográfica y temporal, valores de la media en $\mu^{3}$, desviación standard (s), número de lagunas óseas medidas por muestras $(\mathrm{N})$, y nivel de ploidía resultante $(\mathrm{P})$.

\begin{tabular}{|c|c|c|c|c|c|}
\hline \multirow{2}{*}{$\begin{array}{l}\mathrm{N}^{\mathrm{o}} \text { de colección } \\
\mathrm{MACN} 14324\end{array}$} & \multirow{2}{*}{$\begin{array}{l}\text { Espécie } \\
\text { Ceratophrys prisca }\end{array}$} & \multicolumn{2}{|c|}{$\begin{array}{l}\text { Volumen de laguna } \\
\text { óssea }\left(\mu^{3}\right), \mathrm{s}\end{array}$} & \multirow{2}{*}{$\frac{\mathrm{N}}{20}$} & \multirow{2}{*}{$\begin{array}{l}P \\
2 n\end{array}$} \\
\hline & & 392,00 & $\pm \quad 57,74$ & & \\
\hline MLP 48.XII.16.195 & Ceratophrys sp. & 352,90 & $\pm \quad 69,07$ & 7 & $2 \mathrm{n}$ \\
\hline MACN 14319 & C. prisca var. subcornuta & 790,50 & $\pm 91,36$ & 18 & $2 n$ \\
\hline MACN 14325 & C. prisca var. subcornuta & 687,19 & $\pm 123,91$ & 15 & $4 n$ \\
\hline MACN 14323 & C. prisca var. subcornuta & 282,96 & $\pm \quad 33,32$ & 7 & $2 \mathrm{n}$ \\
\hline MACN 14317 & C. prisca, plesiotipo & 547,03 & $\pm \quad 96,70$ & 8 & $2 n$ \\
\hline MACN 14322 & Ceratophrys sp. & 330,42 & $\pm 79,32$ & 24 & $2 n$ \\
\hline MACN 14318 & C. prisca var. gigantea & 414,61 & $\pm \quad 50,74$ & 16 & $2 \mathrm{n}$ \\
\hline MLP 94.II.1.171 & Ceratophrys sp. & 648,71 & $\pm \quad 82,22$ & 11 & $4 n$ \\
\hline MLP 88.VII.20.1 & Ceratophrys sp. & 418,68 & $\pm 94,54$ & 25 & $2 \mathrm{n}$ \\
\hline MLP 88.VII.20.2 & Ceratophrys sp. & 265,63 & $\pm \quad 75,05$ & 17 & $2 n$ \\
\hline MACN 19731 & Ceratophrys sp. & 318,36 & $\pm \quad 49,89$ & 20 & $2 \mathrm{n}$ \\
\hline MACN 17936 & Ceratophrys sp. & 391,78 & $\pm \quad 36,39$ & 8 & $2 \mathrm{n}$ \\
\hline MLP 34.V.10.8 & Ceratophrys sp. & 133,32 & $\pm \quad 21,90$ & $5 !$ & $2 \mathrm{n}$ ? \\
\hline MACN 18074 & Ceratophrys sp. & 622,23 & $\pm \quad 26,66$ & $3 !$ & $4 n ?$ \\
\hline MACN 17585 & Ceratophrys sp. & 335,70 & $\pm \quad 58,27$ & 21 & $2 \mathrm{n}$ \\
\hline MLP 88.V.20.1 & Ceratophrys sp. & 874,94 & $\pm 185,68$ & 9 & $4 n$ \\
\hline MACN 19744 & Ceratophrys sp. & 765,71 & $\pm 125,05$ & 15 & $4 n$ \\
\hline MLP 86.III.25.151 & Ceratophrys sp. & 621,74 & $\pm \quad 97,79$ & 16 & $4 n$ \\
\hline MLP 86.III.25.150 & Ceratophrys sp. & 1011,93 & $\pm 74,43$ & 9 & $8 \mathrm{n}$ \\
\hline MPL 136 & Ceratophrys sp. & 1269,61 & $\pm 108,90$ & 6 & $8 \mathrm{n}$ \\
\hline MLP 247 & Ceratophrys sp. & 785,27 & $\pm 168,51$ & 55 & $4 n$ \\
\hline MLP 52.IX.27.11 & Ceratophrys sp. & 329,54 & $\pm \quad 67,43$ & 11 & $2 n$ \\
\hline MLP 86.VIII.1.5/6 & Ceratophrys ornata & 794,13 & $\pm 143,87$ & 35 & $4 n$ \\
\hline MLP 86.VIII.1.4 & Ceratophrys ornata & 736,96 & $\pm 160,68$ & 44 & $4 n$ \\
\hline MLP 96.V.18.12 & Ceratophrys sp. & 1045,99 & $\pm 119,34$ & 14 & $8 \mathrm{n}$ \\
\hline BMNH 18895 & C. cornuta? & 593,34 & $\pm 138,03$ & 22 & $4 n$ \\
\hline $\mathrm{S} / \mathrm{n}^{\circ} \mathrm{A}$ & C. ornata & 1056,29 & $\pm 138,02$ & 17 & $8 n$ \\
\hline $\mathrm{S} / \mathrm{n}^{\circ} \mathrm{B}$ & C. ornata & 1136,43 & $\pm 121,46$ & 15 & $8 \mathrm{n}$ \\
\hline $\mathrm{S} / \mathrm{n}^{\circ} \mathrm{A}$ & C. cranwelli & 275,29 & $\pm \quad 35,46$ & 23 & $2 n$ \\
\hline $\mathrm{S} / \mathrm{n}^{\circ} \mathrm{B}$ & C. cranwelli & 284,20 & $\pm \quad 37,01$ & 25 & $2 n$ \\
\hline
\end{tabular}

Este primer registro en material fósil del Ceratophrys de una entidad tetraploide procede del Plioceno, Formación Montehermosense (localidad de Monte Hermoso, Buenos Aires, Argentina) (fig. 2). Esta condición es inexistente en la actualidad. Se comprueba además en este mismo material, la coexistencia de entidades diploides y tetraploides.

También al sur de la provincia de Buenos Aires se hallaron restos de Ceratophrys (Fm. Chapadmalalense) diploides, tanto en Punta Varohué y Punta Lobería como los correspondientes a una nueva forma (Juan Carlos Fernícola, comunic. personal) de la localidad de Miramar, y tetraploides de la localidad de Quequén Salado y posiblemente otro de la localidad de Chapadmalal (tab. I). Este material presenta un color más claro (contrariamente al procedente del Piso Montehermosense de color negruzco) y está mucho menos mineralizado, exigiendo por lo tanto menor trabajo para su procesamiento.

La condición tetraploide aparece nuevamente en pisos más recientes del sudeste y centro-este de la provincia de Buenos Aires (tab. I, figs. 1, 2). El primer registro de 


\section{Volumen}

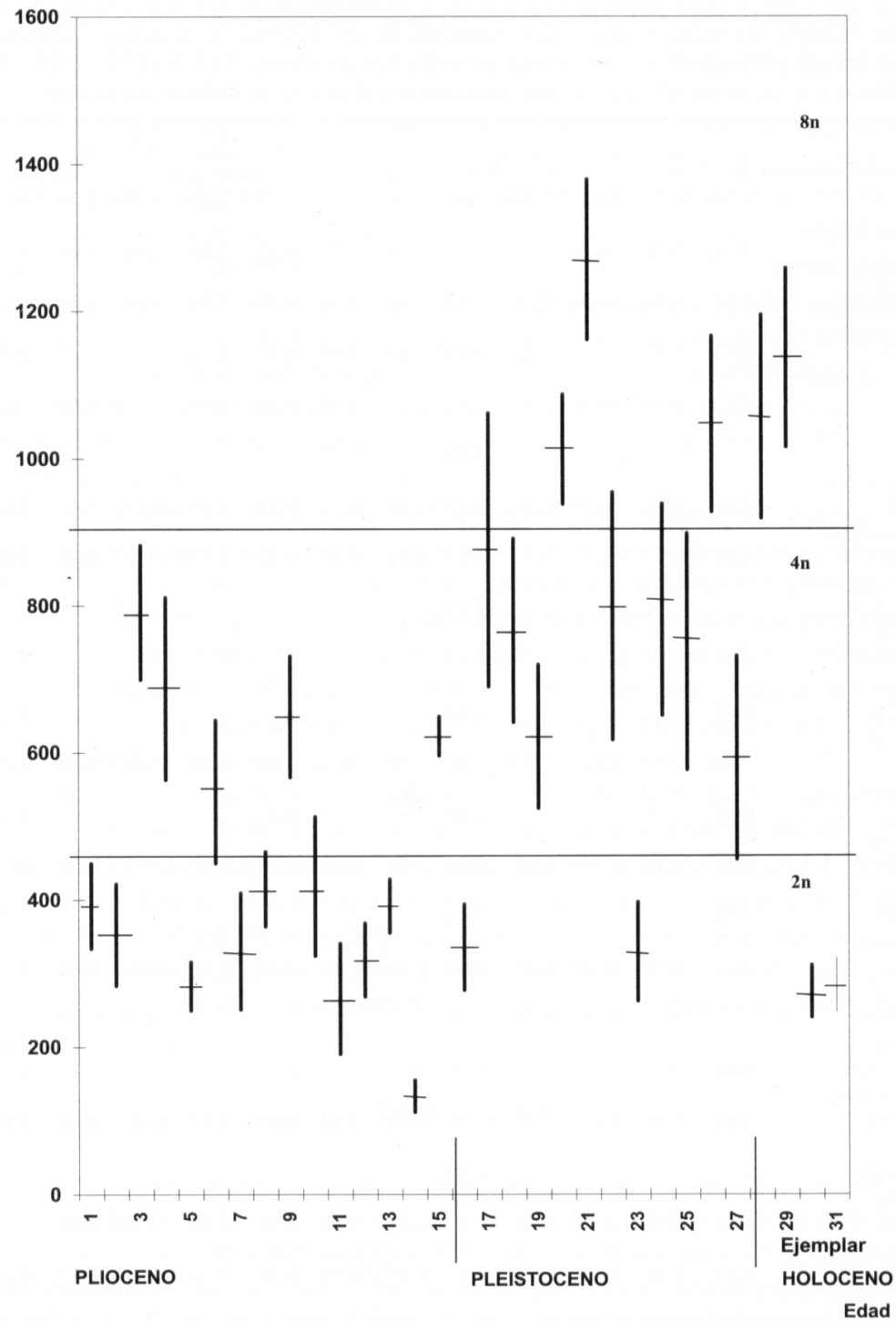

Fig. 1. Representación gráfica del valor de la media y desviación standard del volumen nuclear de las lagunas óseas medidas en fósiles del género Ceratophrys, en correspondencia con el nivel estratigráfico donde fueron hallados. Las líneas horizontales representan el límite de los rangos correspondientes a los distintos niveles de ploidía. (1-8, Fm. Montehermosense; 9, Fm. Chapadmalalense inferior; 10-15, Fm. Chapadmalalense; 16, Fm. Barrancalobense; 17-18, Fm. Ensenadense; 19-22, Pleistoceno; 23, Holoceno superior; 24-26, Pleistoceno tardío-Holoceno; 27, Pleistoceno de Brasil; 28-31, Actual (C. ornata, Buenos Aires, C. cranwelli, Chaco). 
Tabla II. Indices entre ocho caracteres merísticos estudiados en material actual (C. cranwelli y $C$. ornata) y fósil del género Ceratophrys. (1, escotadura postorbitaria/distancia entre las puntas de los escamosales; 2 , distancia entre punta del escamosal y cuadrado/altura del neurocráneo; 3 , ancho del cráneo/ancho forámen magnum; 4 , ancho del cráneo/ancho de la órbita; 5 , distancia interorbital/ancho de la órbita; indices obtenidos de la combinación entre los anteriores $(1 \times 3,1 \times 4,1 \times 2,2 \times 3,3 \times 5,4 \div 3$, $5 \times 1$; P, ploidía). Los números en negrita corresponden a valores de la entidad octoploide.

\begin{tabular}{|c|c|c|c|c|c|c|c|c|c|c|c|c|c|}
\hline Especímenes & 1 & 2 & 3 & 4 & 5 & $1 \times 3$ & $1 \times 4$ & $1 \times 2$ & $2 \times 3$ & $3 \times 5$ & $4 / 3$ & $5 \times 1$ & $\mathrm{P}$ \\
\hline $\begin{array}{l}\mathrm{S} / \mathrm{n}^{\circ} \mathrm{A} \\
\text { Ceratophrys cranwelli }\end{array}$ & 0.23 & 0.72 & 13.29 & 5.53 & 0.90 & 3.06 & 1.27 & 0.17 & 9.57 & 11.96 & 0.42 & 0.21 & $2 n$ \\
\hline $\begin{array}{l}\mathrm{S} / \mathrm{n}^{\circ} \mathrm{B} \\
\text { C. ornata }\end{array}$ & 0.16 & 0.78 & 10.06 & 4.83 & 0.73 & 1.61 & 0.77 & 0.12 & 7.85 & 7.34 & 0.48 & 0.12 & $8 n$ \\
\hline $\begin{array}{l}\mathrm{S} / \mathrm{n}^{\circ} \mathrm{C} \\
\text { C.ornata }\end{array}$ & 0.14 & 0.74 & 10.70 & 4.76 & 0.87 & 1.50 & 0.66 & 0.10 & 7.92 & 9.31 & 0.44 & 0,12 & $8 n$ \\
\hline $\begin{array}{l}\text { MACN } 14319 \\
\text { C. prisca var. subcorn. }\end{array}$ & 0.12 & 0.97 & 12.39 & 5.22 & 0.81 & 1.49 & 0.63 & 0.12 & 12.0 & 10.04 & 0.42 & 0.10 & $4 n$ \\
\hline $\begin{array}{l}\text { MACN } 14325 \\
\text { C.prisca var. subcorn. }\end{array}$ & 0.11 & 0.99 & 10.96 & 5.92 & 0.92 & 1.21 & 0.65 & 0.11 & 10.85 & 10.08 & 0.54 & 0.11 & $4 n$ \\
\hline $\begin{array}{l}\text { MACN } 14323 \\
\text { C. prisca var. subcorn. }\end{array}$ & - & - & - & - & 0.83 & - & & - & - & & & & $2 n$ \\
\hline $\begin{array}{l}\text { MACN } 14317 \\
\text { C. prisca, plesiotipo }\end{array}$ & 0.15 & 0.81 & 10.98 & 5.14 & 0.85 & 1.65 & 0.77 & 0.12 & 8.89 & 9.33 & 0.47 & 0.13 & $4 n$ \\
\hline $\begin{array}{l}\text { MACN } 14318 \\
\text { C. prisca var. gigantea }\end{array}$ & 0.14 & 0.93 & 12.21 & 5.73 & 0.92 & 1.71 & 0.80 & 0.13 & 11.36 & 11.23 & 0.47 & 0.13 & $2 n$ \\
\hline $\begin{array}{l}\text { MLP 94.II.1.171 } \\
\text { Ceratophrys sp. }\end{array}$ & - & - & - & - & 0.84 & - & & - & - & & & & $4 n$ \\
\hline $\begin{array}{l}\text { MLP 88.VII.20. } 1 \\
\text { Ceratophrys sp. }\end{array}$ & 0.18 & & & & 1.10 & & & & & & & 0.20 & $2 n$ \\
\hline $\begin{array}{l}\text { MLP 88.VII. } 20.2 \\
\text { Ceratophrys } \quad \mathrm{sp} .\end{array}$ & 0.12 & - & - & - & 0.88 & & & & & & & 0.11 & $2 n$ \\
\hline $\begin{array}{l}\text { MPL } 136 \\
\text { Ceratophrys sp. }\end{array}$ & 0.16 & 0.77 & 10.65 & 4.73 & 0.90 & 1.70 & 0.76 & 0.12 & 8.20 & 9.59 & 0.44 & 0.14 & $8 n$ \\
\hline $\begin{array}{l}\text { MLP } 247 \\
\text { Ceratophrys sp }\end{array}$ & 0.14 & 0.76 & - & - & 0.89 & - & & 0.11 & - & & & 0.12 & $4 n$ \\
\hline $\begin{array}{l}\text { MACN } 19731 \\
\text { Ceratophrys sp. }\end{array}$ & 0.16 & 0.82 & 11.91 & 5.06 & 0.89 & 1.91 & 0.81 & 0.13 & 9.77 & 10.56 & 0.42 & 0.14 & $2 n$ \\
\hline $\begin{array}{l}\text { MLP 88.V.20.1 } \\
\text { Ceratophrys sp. }\end{array}$ & 0.18 & - & - & - & 1.10 & - & & - & - & & & 0.20 & $4 n$ \\
\hline $\begin{array}{l}\text { MACN } 19744 \\
\text { Ceratophrys sp. }\end{array}$ & 0.22 & 0.85 & 11.00 & 5.29 & 0.86 & 2.42 & 1.16 & 0.19 & 9.35 & 9.46 & 0.48 & 0.19 & $4 n$ \\
\hline $\begin{array}{l}\text { MLP 52.IX.27.11 } \\
\text { Ceratophrys sp. }\end{array}$ & - & - & 17.88 & 7.55 & 1.05 & - & & - & - & 18.77 & 0.42 & - & $2 n$ \\
\hline $\begin{array}{l}\text { MLP 86.VIII.1.5/6 } \\
\text { Ceratophrys ornata }\end{array}$ & 0.15 & - & - & - & 0.79 & - & & - & - & & & 0.12 & $4 n$ \\
\hline $\begin{array}{l}\text { MLP 86.VIII.1.4 } \\
\text { C. ornata }\end{array}$ & 0.12 & 0.76 & 9.22 & 5.32 & 0.91 & 1.11 & 0.64 & 0.09 & 5.53 & 8.40 & 0.58 & 0.11 & $4 n$ \\
\hline $\begin{array}{l}\text { MLP 96.V.18.12 } \\
\text { Ceratophrys sp. }\end{array}$ & - & - & - & - & 0.98 & - & & - & - & & & & $8 n$ \\
\hline $\begin{array}{l}\text { BMNH } 18896 \\
\text { C. cornuta }\end{array}$ & 0.13 & 0.82 & 11.20 & 6.46 & 1.20 & 1.47 & 0.84 & 0.11 & 9.18 & 13.44 & 0.58 & 0.16 & $?$ \\
\hline $\begin{array}{l}\text { BMNH } 18896 \mathrm{a} \\
\text { C. cornuta }\end{array}$ & 0.13 & 0.81 & 9.80 & 5.05 & 0.87 & 1.27 & 0.72 & 0.11 & 7.94 & 8.53 & 0.52 & 0.11 & $?$ \\
\hline
\end{tabular}

octoploidía en material fósil corresponde a material procedente del partido de Gral. La Madrid, Piso Holoceno temprano-medio.

En varios casos el número de mediciones fue reducido, oscilando entre 6 y 18 por preparado, debido a que la orientación de los osteoclastos y/o la densidad de los mismos en el pequeño trozo de hueso extraído no fuera la ideal; no obstante en otros preparados 
se alcanzó a contar hasta 55 lagunas óseas. Se observa que los valores de desviación standard son bastante elevados lo que demuestra una gran variación del tamaño individual de las lagunas, como también ocurre en especies vivientes de Xenopus. Se verifica cierta particularidad en las variaciones individuales: los organismos diploides son los que presentan menor desviación respecto a la media cualquiera sea su procedencia como antigüedad y los tetraploides, se advierte la tendencia hacia desviaciones mayores respecto de la media (fig.1, tab. I). Esto también se observa en los octoploides. Se registraron asimismo algunos casos de mayor desviación standard en tetraploides.

Se destaca en los tetraploides del Plioceno o Pleistoceno inferior que los valores individuales eran más uniformes que en los procedentes del Pleistoceno superior, los que mostraban más frecuentemente valores extremos, siendo el más significativo un ejemplar (MLP 247) de Monte Hermoso, cuya edad lamentablemente no estaba indicada. En los octoploides algunos valores de volumen de laguna ósea sobrepasan en mucho al valor de la media. Los volúmenes de las lagunas óseas que excedían grandemente a los mayores valores promedio de cada preparado fueron descartados, pero no menospreciados en las consideraciones finales.

Se observa que en los ejemplares del Plioceno inferior de Monte Hermoso, determinados como diploides, la mayoría de sus índices morfométricos se aproximaban a los de $C$. cranwelli actuales, coincidiendo además y casualmente con su condición diploide. Tal es el caso de C. prisca var. gigantea que se destaca por sus grandes dimensiones. Lo más llamativo de su cráneo, además de su tamaño es el hecho que la rama ótica del escamoso sobrepasa en mucho a los cóndilos y sus puntas se dirigen levemente hacia arriba. Los tetraploides muestran un predominio de índices cercanos a los de la octoploide $C$. ornata.

A pesar de no contar con la totalidad de los índices de los tetraploides del piso Ensenadense (hallados al norte de Mar del Plata), curiosamente el índice 1 (tab. II) se aproxima al de la especie diploide, índice muy relevante para este estado. Los ejemplares del Pleistoceno de Brasil, de acuerdo a los índices calculados, se trataría de dos entidades diferentes. Lamentablemente no se tienen los datos de su nivel de ploidía ya que sólo se nos proporcionó material óseo de un tercer ejemplar, que resultó ser tetraploide. Tentativamente se puede decir que entre ellos habría un ejemplar octoploide, tal como lo revelarían sus índices.

\section{DISCUSIÓN}

Podría cuestionarse la validez del método de determinación del nivel de ploidía en material fósil a través de la medición de los osteoclastos, sin embargo se puede sostener que es útil y valedero. De acuerdo a recientes estudios realizados sobre la diagénesis en fósiles pleistocenos (PFRETZSCHNER, 2000) la principal deposición de pirita que se produce en los conductos óseos sería consecuencia de la degradación de hemoglobina $\left(\mathrm{Fe}^{2+}\right)$ y colágeno $\left(\mathrm{S}^{2-}\right)$ a nivel de la médula ósea y se restringiría a las zonas cercanas a ésta. Por subsecuente oxidación de la pirita (tardíamente en el proceso diagenético) se produce ácido sulfúrico que podría ensanchar los conductos óseos, tales como los conductos de Havers y lagunas óseas. A lo recién expuesto se puede comentar que si bien no fue realizado ningún estudio para comprobar la existencia del contenido de pirita de las lagunas óseas, éste debió haber sido escaso ya que los trozos de huesos utilizados 


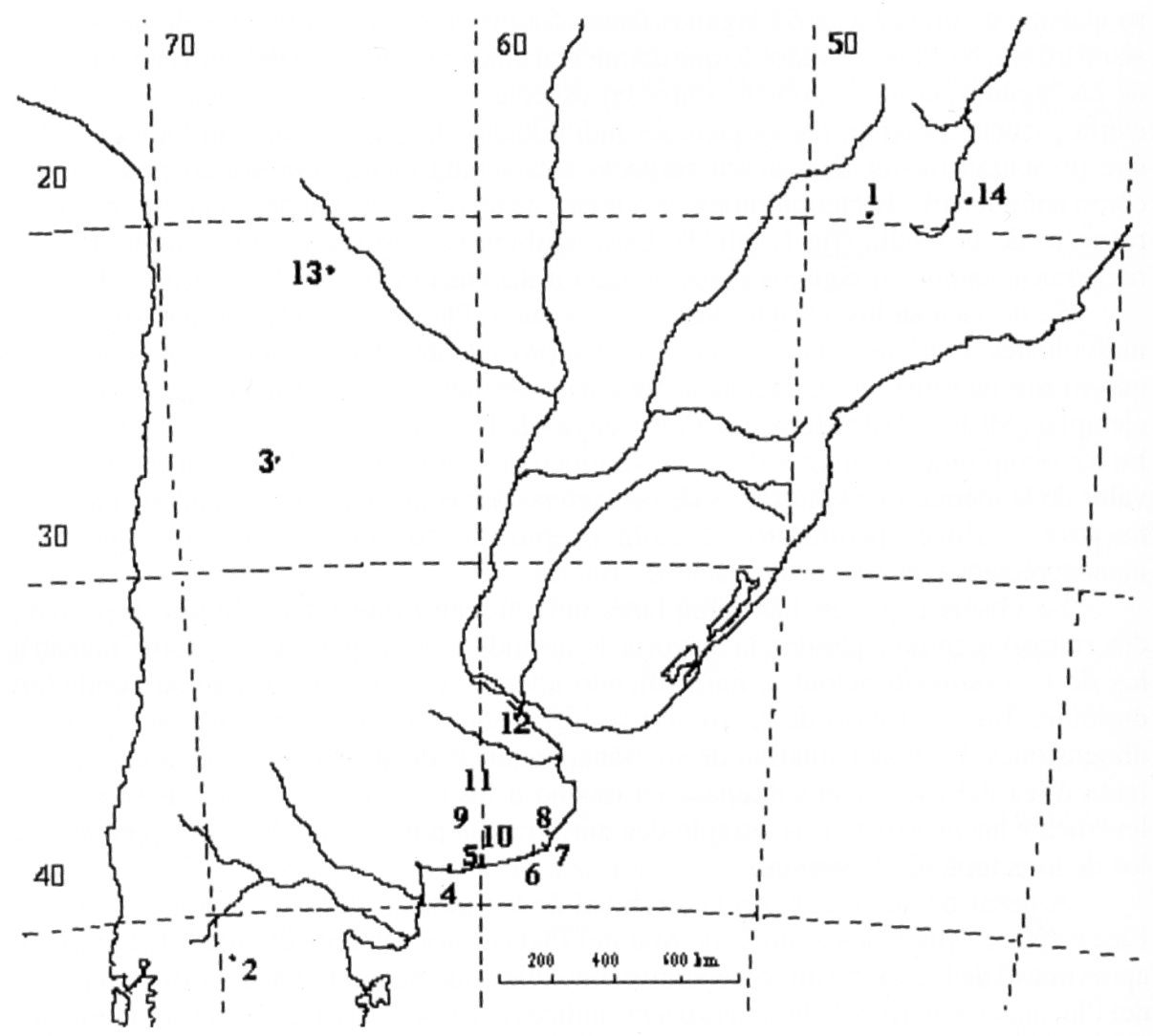

Fig. 2. Ubicación geográfica del material fósil descubierto hasta el presente, correspondiente al género Ceratophrys. Cretácico superior: 1, Uberaba (Brasil); Mioceno: 2, Ing. Jacobacci, Rio Negro; Plioceno: 3, Corral Quemado, Catamarca; 4, Monte Hermoso, Bs.As.; 5, Quequén Salado, Bs.As.; 6, Miramar, Bs.As.; 7, Chapadmalal, Bs.As.; Pleistoceno: 8, Mar del Plata, Bs.As.; 9, Gral. La Madrid, Bs.As.; 10, González Chávez, Bs.As.; 11, Olavarría, Bs.As.; 12, Ensenada, Bs.As.; 14, Tarija (Bolivia); 16, Lagoa Santa, Minas Gerais (Brasil).

procedían de huesos craneanos planos, descartando una abundante provisión endógena (medular) de azufre y hierro. Esto minimizaría la ulterior producción de ácido sulfúrico. Las determinaciones de volumen de las lagunas óseas efectuadas en material pliocénico evidenciaron la coexistencia de entidades diploides y tetraploides del mismo yacimiento. Si las lagunas óseas del material fósil más antiguo aquí estudiado hubiesen sufrido la acción del ácido sulfúrico en alguna de las etapas diagenéticas, hubiese sido imposible la detección de entidades diploides en yacimientos pliocenos.

Debido a la distinta denominación de las edades de acuerdo a las áreas geográficas involucradas, fueron consultados Ameghino (1909), Kraglievich (1952), PASCuAL (1966), Tonni \& Fidalgo (1978), Báez \& Yané (1979), Cione \& Tonni (1995a, b; 1996,1997).

El resto más antiguo de esta subfamilia, Ceratophryinae (tal como es considerado para este grupo de batracios por Frost, 1985) correspondería a W. gerholdi (Mioceno 
medio de Ingeniero Jacobacci, noroeste de Río Negro, Argentina) (fig. 2), afín al género Ceratophrys, tal como lo han aseverado CAsamiquela (1963) y BÁez \& Perí (1989) principalmente por las características de su esqueleto postcraneano y por el cráneo con saliencia del escamosal típicamente angular e hiperosificado. Llama la atención la proporción cabeza-cuerpo, característica atribuíble a un individuo juvenil (BÁez \& PeRí, 1990).

Recién en el Plioceno tardío se vuelve a tener registros de fósiles de esta subfamilia al sur de la provincia de Buenos Aires (Monte Hermoso, Fm. Montehermosense). Los hallazgos fueron hechos por primera vez por AMEGHINo (1899), quien los designó como C. prisca. Rovereto (1914) distingue tres variedades: C. p. subcornuta, C. p. gigantea y C. p. prisca, correspondiente a material encontrado en el mismo nivel estratigráfico que C. prisca. Se registraron tanto entidades diploides como tetraploides. Este material se caracteriza por su color negruzco. Comúnmente los huesos fósiles procedentes de depósitos marinos poseen una coloración negra; tal característica no es hallada en depósitos fluviales ya que el contenido de sulfuros de las aguas es bajo (PFrETzSCHNER, 2000). Esto sugeriría que la fosilización de dicho material tuvo lugar durante una transgresión marina.

De acuerdo a los resultados obtenidos del análisis del nivel de ploidía, índices morfométricos y morfología craneana, concordaríamos con BÁEZ \& GASPARINI (1977) y PERí (1993) en invalidar las tres variedades, incluso de la consideración uniespecífica de estas especies. Procede de la misma formación un ejemplar hallado en Corral Quemado (Catamarca, Argentina) (Marshall \& Patterson, 1981). En el Plioceno se vuelven a encontrar al sur de la prov. de B. Aires, en el Piso Chapadmalalense, ejemplares diploides (Miramar, Pta. Lobería y Pta. Varohué) y tetraploides (Quequén Salado y Chapadmalal). En el Piso Barrancalobense, terminando el Plioceno, se registró un fósil diploide.

Ya en el Pleistoceno, y siguiendo la franja de costa atlántica, otros restos fósiles de anuros asignados a Ceratophrys (Piso Ensenadense) fueron hallados a $1 \mathrm{~km}$ y a $18 \mathrm{~km}$ (este último colectado por los autores) al norte de Mar del Plata (fig. 2). Los ejemplares presentaban la misma coloración marrón claro y el escudo de hueso dérmico del cráneo no llegaba, visto dorsalmente, a cubrir los cóndilos. Se observa una diferencia entre ambos, en uno de ellos (18 km al N) el canal para la arteria carótida se encuentraba ubicado a cierta distancia del escudo craneano y no inmediatamente por debajo, como en el resto de los ejemplares, incluyendo los actuales. Esta misma característica fue encontrada en un ejemplar de la formación La Postrera, Pleistoceno tardío-Holoceno. Las mediciones de las lagunas óseas sugieren que se tratarían de organismos tetraploides en ambos casos.

Del Piso Ensenadense también procede material que Rusconi (1932) asigna a $C$. ensenadensis de Olivos, B. Aires, Argentina, no examinado, siendo lo más probable que se trate de C. ornata (Reig, 1958). Ameghino (1899) también señala C. ornata en los alrededores de B. Aires.

Del Pleistoceno tardío-Holoceno, el material estudiado procedente de Miramar (Holoceno superior, antes Fm. Aimarense (Eduardo P. Tonni, comunic. personal) resultaría ser diploide al igual que los del Chapadmalalense anteriromente citados. Perí (1993) estudia material procedente de Laguna de Los Tres Reyes en el centro-sur de la prov. de B. Aires (González Chávez), Fm. La Postrera. Los dos ejemplares estudiados resultaron ser tetraploides, lo cual refutaría la asignación de PERí (1993) de este material a C. ornata. Uno de ellos tenía la particularidad de que el canal para la arteria carótida tenía la misma 
posición que en el tetraploide de la Fm. Ensenadense hallado a $18 \mathrm{~km}$ al norte de Mar del Plata. En todo este material el escudo óseo craneano apenas cubría los cóndilos, visto dorsalmente. El material óseo se caracterizaba por su aspecto de hueso proveniente de un animal más reciente y además era más duro, no tan frágil como en los casos anteriores exceptuando, como fuera ya mencionado, los del Montehermosense. El tamaño del foramen magnum es ciertamente más grande que en el resto de los ejemplares fósiles estudiados, y el escamoso menos desarrollado.

VergnaUd-Grazzini (1968) describió material perteneciente a Ceratophrys de las localidades de Ñuapua (región chaqueña) (fig.2) y Tarija (región andina) del Pleistoceno superior de Bolivia. De la misma edad proceden restos fósiles de Brasil (Lagoa Santa, Minas Gerais) (fig.2) que GüNTHER (1859) asignó a C. cornuta (BMNH). El material examinado resultó tener un nivel de ploidía correspondiente a un organismo tetraploide.

En el Holoceno temprano se destaca en Tapalqué (Olavarría, B. Aires), un ejemplar tetraploide y otro octoploide, y en Fortín Necochea (Gral. La Madrid, B. Aires) de uno octoploide. Es decir que recién en el Holoceno temprano a medio podemos aventurarnos a decir, considerando el escaso material disponible, que hace su aparición la condición octoploide.

En relación a las condiciones paleoambientales imperantes en los períodos correspondientes a los fósiles considerados, con anterioridad a $W$. gerholdi, existen evidencias de que durante el Paleoceno tardío-Eoceno temprano, los climas globales eran relativamente cálidos, atemperándose hacia el Eoceno tardío (PASCUAL, 1984; BÁEZ, 1986). Esta situación se prolongaría hacia el Oligoceno. Incluso durante el Mioceno temprano, los mamíferos hallados indicarían la existencia de condiciones ambientales cálidas (Pascual \& Rivas, 1971; Pascual, 1984). Esto permitió la presencia de una biota característica de climas subtropicales en la Patagonia, lo cual reforzaría lo aseverado por LYNCH (1971), Estes \& REIG (1973) y BÁEZ \& GASPARINI (1979) entre otros, quienes sostienen que varias familias de anfibios anuros (hílidos, leptodactílidos y bufónidos) tuvieron su origen en este continente dispersándose por el mismo de acuerdo a las distintas condiciones climáticas imperantes, alcanzando distintos grados de diferenciación y/o especialización. Wawelia gerholdi habitó en territorio patagónico (Ing Jacobacci, Rio Negro) durante el Mioceno medio, época en la cual comenzó el levantamiento del macizo patagónico (Pascual, 1984). De acuerdo a Casamiquela (1963), el elenco de diatomeas asociado a Wawelia es indicador de un clima templado a frío. Es decir que ya se haría sentir el cambio climático producido tanto por la elevación de la cordillera patagónica (Mioceno tardío), que frenaría los vientos húmedos del Pacífico incrementando paulatinamente las condiciones áridas de la zona, como la apertura del pasaje de Drake y la formación de la corriente circumpolar antártica, trayendo como consecuencia la modificación de patrones de circulación atmosférica y oceánica (BÁEZ, 1986). A esto se sumaría la impresionante transgresión marina (Mioceno medio al Plioceno) que ocupó las zonas bajas y precordilleranas del continente sudamericano (NE de la Patagonia, llanura Chaco-pampeana y Mesopotamia (del territorio argentino), Paraguay, Bolivia, Norte de Perú, Colombia y Venezuela) (RäsäNEN et al., 1995).

Durante el Plioceno temprano y medio el norte de la Patagonia el clima era más húmedo que el actual, cambiando a más seco y frío en el Plioceno tardío. Biológicamente es muy significativo ya que la Patagonia pasó a ser de extraordinario centro de evolución (y probablemente también de dispersión) (PASCUAL \& RIVAS, 1973) a convertirse en un territorio cada vez más árido. Esto obligó a que la fauna migrase hacia el norte como 
reflejo de ese deterioro, o que las más euritérmicas se dirigieran hacia áreas más húmedas del oeste de los Andes (BÁEZ \& GASPARINI, 1977).

Ya en el Plioceno (Fm. Montehermosense) se registra la presencia de Ceratophrys en el noroeste del país (Catamarca) y en el sur de la prov. de B. Aires: a) en la región subandina la fauna registrada en este período poseía un marcado carácter subtropical. Esto hace inferir que las condiciones ambientales eran similares a aquéllas del oeste del distrito chaqueño (ámbito de la actual distribución de la especie diploide en el territorio argentino) que a su vez, aparentemente, también existieron en el sudoeste de la prov. de B. Aires (BÁEZ \& YANÉ, 1979). La culminación del levantamiento de las Sierras Pampeanas que se registra en el Plioceno tardío, habría impedido el paso de los vientos húmedos del nordeste provocando la desertización de las áreas occidentales (PASCUAL \& RIVAS, 1973). Lamentablemente no se pudo estudiar material de Ceratophrys de Catamarca, el cual hubiese aportado una valiosa información, no sólo determinando su nivel de ploidía y poder esbozar hipótesis sobre su posible procedencia, sino también para las observaciones realizadas por MERCADAL (1981). Este autor (1986) destacó el hecho de que los índices craneanos de la especie octoploide, descripta para el nordeste del Brasil, C. joazeirensis Mercadal, 1986, se aproximan más a los de C. cranwelli del norte de la Argentina; b) el clima del sudoeste de la prov. de B. Aires se caracterizó por presentar estaciones con diferente régimen de lluvias (TonNI, 1974; BÁEZ \& GASPARINI, 1977). Luego con el ascenso de la región montañosa andina (Plioceno-Pleistoceno) hizo que prevaleciera el clima seco provocando a su vez una conexión faunística entre el sudoeste de B. Aires y Patagonia, y una expansión hacia el este de formas provenientes del oeste (BÁEZ \& YANÉ, 1979). Esto pudo haber favorecido la expansión del bosque xerófilo, el cual está presente en la llanura pampeana hacia el oeste y norte (BÁEz \& YANÉ, 1979; ToNNI \& FIDALGO, 1978), y la consecuente retracción de la distribución de las formas diploides y tetraploides del territorio bonaerense, como pudo ser verificado en el presente trabajo.

Tres hechos son llamativos: el primero, referido al diploides al sur de la prov. de B. Aires, territorio exclusivo en la actualidad para la entidad octoploide, avalando lo aseverado por Tonni (1974), Ringuelet (1978), Gallardo (1979) y Pascual (1984), que durante la Edad Montehermosense, la distribución de algunos elementos de la fauna subtropical o del "tipo chaqueño" se extendía hasta el sur de la prov. de B. Aires. Segundo, la existencia en tiempos pretéritos de la contraparte tetraploide, ausente hoy en día, y tercero, la coexistencia de estas dos formas durante este período y posteriores.

Partiendo de los datos obtenidos del material estudiado y del panorama paleoclimático recién expuesto se observa que existiría prima facie una estrecha relación entre las fluctuaciones climáticas y la evolución del nivel de ploidía en Ceratophrys. Se visualiza además un "movimiento" sudoeste-nordeste, correspondiente a la sucesión Plioceno-Holoceno, del incremento del nivel de ploidía para los ejemplares de Ceratophrys en la prov. de B. Aires. No se ha registrado ningún hallazgo de material del Plioceno para el norte de esta provincia. El último registro para la forma diploide está indicado para el Holoceno Superior, siendo para la forma tetraploide las postrimerías del Pleistoceno (con la coexistencia de octoploides). Lamentablemente no se posee la ubicación cronológica certera del material tetraploide y octoploide de Monte Hermoso (MLP 247, 136), supuestamente del Pleistoceno.

Los organismos diploides son los que presentan menor desviación respecto a la media cualquiera sea su procedencia como antigüedad, advirtiendo en los 
tetraploides la tendencia hacia desviaciones mayores respecto de la media, lo cual es observado también en los octoploides. Esto concordaría con los resultados obtenidos por Reumer \& THiÉBAud (1987) en Xenopus, quienes destacan igualmente el incremento de la desviación standard a medida que se incrementa el nivel de ploidía. Pero a diferencia de los resultados de dichos autores, si bien hubo un incremento de dicho parámetro con el mayor grado de ploidía, se registraron algunos casos de mayor desviación standard en ejemplares tetraploides. El hecho de que los tetraploides pliocénicos hayan sido los que presentaran un conjunto de valores individuales más regulares que los pleistocénicos (por lo tanto de menor desviación standard) hablan de cierta estabilidad genética, por lo menos en cuanto al contenido nuclear de ADN.

Cambios climáticos pudieron haber forzado a estos batracios a emigrar hacia el norte buscando condiciones más propicias de humedad y temperatura. Estas nuevas condiciones pudieron haber tenido ingerencia en el establecimiento de una nueva situación en el patrimonio genético. Tal vez el indicio más evidente de esta transición haya sido la presencia en tetraploides más recientes (pleistocenos) de valores de desviación standard mucho más elevados, reflejando una tendencia a un cambio del contenido nuclear de ADN (predominantemente hacia uno mayor). Un ejemplo de ello es la presencia en Monte Hermoso de un ejemplar 4n y otro 8n, ambos del Pleistoceno, teniendo el primero uno de los mayores valores de desviación standard entre los tetraploides determinados.

La ausencia actual de Ceratophrys tetraploides sugeriría que tal irregularidad en los valores de los tetraploides pleistocénicos haya sido reflejo de la falta de adaptación (o inestabilidad genética) a las nuevas condiciones y adopción de un nuevo estado, el octoploide, característico para este género en la llanura bonaerense actual. Se han registrado casos de simpatría con diploides al sur de la provincia de B. Aires. Inicialmente una especie poliploide debería acompañar simpátricamente a la población diploide ancestral (BOGART \& WASSERMAN, 1972), adquiriendo subsecuentemente mecanismos de aislamiento reproductivo. Análogamente suponemos que habría ocurrido lo mismo entre las poblaciones tetraploide y octoploide.

GoIN \& GoIN (1968) exponen claramente la relación entre el contenido de ADN por núcleo y el grado de avance evolutivo, observando que en los vertebrados primitivos, o los más primitivos de cada clase, el contenido de ADN es mucho mayor que los organismos que han alcanzado la máxima especialización, como por ejemplo, las aves y los peces teleósteos. De esta forma un grupo con ADN redundante conserva la suficiente plasticidad evolutiva para producir importantes cambios adaptativos requeridos en la evolución cuántica. Así el tipo 3 de evolución postulado por RENDEL (1965) que involucra el incremento de material genético (por poliploidía, entre otros mecanismos) posibilitaría la aparición de novedades evolutivas manteniendo, mientras, las más viejas. La poliploidía proveería además de un mayor número de posibles combinaciones genéticas como consecuencia de la segregación independiente de homólogos multivalentes (BЕÇAK et al.,1970). Es decir que la "nueva" adquisición del estado poliploide sería recién el comienzo de una etapa evolutiva en este taxón.

Por el análisis del genoma de numerosos organismos y comparando varias familias génicas, se ha propuesto que en la evolución de los vertebrados pudo haberse producido, 
ya muy tempranamente (hace millones de años), dos duplicaciones genómicas, resultando una octoploidización (SPRING, 1997; GIBSON \& SPRING, 2000). El genoma de los vertebrados es mayor que el de los invertebrados y muestra evidencias de duplicación génica. Se barajarían dos posibilidades, pudo haberse producido una tetraploidización seguida por un proceso de diploidización durante varios millones de años y luego una segunda tetraploidización, o una rápida octoploidía ocurrida en pocos cientos o miles de años. Tal sería el caso de las plantas de cultivo y de la especie de anfibios Ceratophrys (GIBson \& SPRING, 2000).

Un estudio más profundo merecerían los ejemplares provenientes del Pleistoceno de Brasil. Tanto la morfología craneana como los índices obtenidos de este material dan idea de la coexistencia de dos distintas entidades y, al igual que en Argentina, en la actual zona de distribución de la especie octoploide (C. aurita), habría habitado una forma tetraploide. Más al norte, en Pernambuco, se encuentra $C$. joazeirensis entidad octoploide que se caracteriza por su morfología y morfometría similar a $C$. cranwelli (diploide) del norte de Argentina y Bolivia, y no a C. aurita (octoploide), a pesar de la mayor aproximación geográfica. Se podrían esbozar distintas hipótesis para poder dilucidar la historia de este mecanismo evolutivo, pero la información disponible es muy incompleta. Otro material que podría estar vinculado a los ceratofrinos y cuyo estudio podría aportar una valiosa información por su localización geográfica es Baurubatrachus pricei, descripta por BÁEz \& PeRí (1989) del Cretácico superior hallado al oeste de Minas Gerais.

Vialli \& Sacchi-Vialli (1969) destacaron las importantes implicaciones que tiene el análisis de las lagunas óseas para el estudio de la evolución de los vertebrados. Por lo tanto no habría dudas que este método de determinación del nivel de ploidía en ejemplares fósiles constituiría de por sí una valiosa herramienta para el estudio evolutivo de determinados grupos animales (vegetales?) abriendo la posibilidad (complementado con otros estudios) de barajar nuevas hipótesis sobre su significado y los factores intervinientes en su establecimiento.

Agradecimientos. A las instituciones y a sus investigadores que nos han facilitado el material para estudio: Alejandro Kramarz (MACN), Rosendo Pascual, Marcelo Reguero, Susana Bargo (MLP) y Sandra Chapman (BMNH); a José Bonaparte (MACN) por todo su apoyo, a Ana María Báez (Facultad de Ciencias Naturales, Universidad de Buenos Aires) por la revisión crítica del manuscrito y a Eduardo P. Tonni (MLP) por la valiosa información de índole estratigráfica suministrada.

\section{REFERENCIAS BIBLIOGRÁFICAS}

Ameghino, F. 1899. Sinopsis geológico-paleontológica. Suplemento. La Plata. 13p.

1909. Formaciones sedimentarias de la región litoral de Mar del Plata y Chapalmalán. An. Mus. nac. B. Aires, Buenos Aires, 10(3):343-429.

Báez, A. M. 1986. El registro terciario de los anuros en territorio argentino: una reevaluación. In: Congreso Argentino de Paleontologia y Bioestatigrafia, 4º Mendoza, 1986, Actas... Mendoza, Inca. v.2, p.107-118.

BÁez, A. M. \& Gasparini, Z. B. DE. 1977. Orígenes y evolución de los anfibios y reptiles del Cenozoico de América del Sur. Acta geol. lilloana, Tucumán, 14:149-232.

1979. The South American herpetofauna: an evaluation of the fossil record. In: Duellman, W. E. ed. The South American Herpetofauna: Its origin, evolution, and dispersal. The University of Kansas. p. 29-54. (Monograph $\mathrm{n}^{\circ}$ 7)

BÁez, A. M. \& Perí, S. 1989. Baurubatrachus pricei, nov. gen. et nov. sp., un anuro del Cretácico superior de Minas Gerais, Brasil. Anais Acad. bras. Cienc., Rio de Janeiro, 61(4):447-458. 
1990. Revisión de Wawelia gerholdi, un anuro del Mioceno de Patagonia. Ameghiniana, Buenos Aires, 27(3-4):379-386.

BÁEz, A. M. \& YANÉ, G. J. S. 1979. Late cenozoic environmental changes in temperate Argentina. In: Duellman, W. E. ed. The South American Herpetofauna: Its origin, evolution, and dispersal. The University of Kansas. p. 141-156. (Monograph $\mathrm{n}^{\mathrm{o}} 7$ )

BARrio, A. 1980. Una nueva especie de Ceratophrys (Anura, Ceratophryidae) del Dominio Chaqueño. Physis, Buenos Aires, 39(96):21-30.

Beçak, M. L.; Denaro, L. \& Beçak, W. 1970. Polyploidy and mechanisms of karyotypic diversification in Amphibia. Cytogenetics, Basel, 9:225-238.

BOGaRT, J. P. 1980. Evolutionary implications of polyploidy in amphibians and reptiles. In: LewIS, W. H. ed. Polyploidy. Biological relevance. Basic life science. New York, Plenum. v.13, p.341-377.

Bogart, J. P. \& Wasserman, A. O. 1972. Diploid-polyploid cryptic species pairs: a possible clue to evolution by polyploidization in anuran amphibians. Cytogenetics, Basel, 11:7-24 .

Casamiquela, R. 1963. Sobre un par de anuros del Mioceno de Río Negro (Patagonia), Wawelia gerholdi n. gen. et sp. (Ceratophrydidae) y Gigantobatrachus parodii (Leptodactylidae). Ameghiniana, Buenos Aires, 3:141-160.

Cione, A. L. \& Tonni, E. 1995a. Los estratotipos de los pisos Montehermosense y Chapadmalalense (Plioceno) del esquema cronológico sudamericano. Ameghiniana, Buenos Aires, 32(4):369-374.

1995b. Chronostratigraphy and "Land-Mammal Ages" in the Cenozoic of Southern South America: principles practices, and the "Uquian" problem. J. Paleont., Tulsa, 69(1):135-159.

1996-1997. Biostratigraphy and chronological scale of uppermost Cenozoic in the Pampean Area, Argentina. In: Rabassa, J. \& Salemme, M. eds. Quaternary of South America and Antarctic Peninsula. Balkema. v.12, p.23-51.

Estes, R. 1975. Fossil Xenopus from the Paleocene of South America and the zoogeography of pipid frogs. Herpetologica, Chicago, 31(3):263-278.

Estes, R. \& Reig, O. A. 1973. The early record of frogs: a review of the evidence. In: VIAL, J. L. ed. Evolutionary biology of the anurans. Contemporary research on major problems. Columbia, Univ. of Missouri. p.11-63.

Frankhauser, G. 1945. Chromosome number and development. Q. Rev. Biol., Baltimore, 20:20-78.

Frost, D. R. 1985. Amphibian species of the world. A taxonomic and geographical reference. Lawrence, Allen \& Association of Systematics Collections. 732p.

Gallardo, J. M. 1979. Composición, distribución y origen de la herpetofauna chaqueña. In: Duellman, W.E. ed. The South American Herpetofauna: Its origin, evolution, and dispersal. The University of Kansas, p.299-307. (Monograph $\mathrm{n}^{\circ}$ 7)

George, S. A. \& Lennartz, M. R. 1980. Methods for determining ploidy in amphibians: nucleolar number and erythrocyte size. Experientia, Basel, 36:687,688.

Gibson, T. J. \& Spring, J. 2000. Evolution of sequences, structures and genomes. Evidence in favour of ancient octaploidy in the vertebrate genome. Biochem. Soc. Transactions, London, 28:259264.

Goin, O. B. \& GoIn, C. J. 1968. DNA and the evolution of the vertebrates. Am. Midl. Nat., Notre Dame, 80(2):289-298.

GüntheR, A. 1859. On sexual differences found in bones of some recent and fossil species of frogs and fishes. Ann. Mag. nat. Hist., London, 3:377-386.

GüNTHER, R. 1977. Die erythrozytengröße als kriterium zur unterscheidung diploider und triploider Teichfrösche, Rana "esculenta" L. (Anura). Biol. Zbl., Leipzig, 96:457-466.

Kraglievich, J. L. 1952. El perfil geológico de Chapadmalal y Miramar, Provincia de Buenos Aires. Revta Mus. munic. Cienc. nat. Mar del Plata, Mar del Plata, 1:8-37.

LYNCH, J. D. 1971. Evolutionary relationships, osteology, and zoogeography of the leptodactyloid frogs. Misc. Publ. Mus. nat. Hist. Univ. Kans., Lawrence, 53:1-238.

Macgregor, H. C. \& Uzzel, T. M., JR. 1964. Gynogenesis in salamanders related to Ambystoma jeffersonianum. Science, New York, 143:1043-1045.

Marshall, L. G. \& Patterson, B. 1981. Geology and geochronology of the mammal-bearing Tertiary of the Valle de Santa María and río Corral Quemado, Catamarca province, Argentina. Fieldiana, Geol., Chicago, 9:1-80.

Mercadal, I. T. 1981. Determinación del nivel de ploidía en ejemplares preservados del género 
Ceratophrys. Amphibia-Reptilia, Leiden, 1(3/4):205-212.

1986. Ceratophrys joazeirensis sp. n. (Ceratophryidae, Anura) del nordeste de Brasil. AmphibiaReptilia, Leiden, 7(4):313-334.

Morescalchi, A. 1977. Adaptation and karyotype in Amphibia. Boll. Zool., Napoli, 44:287-294.

Pascual, R. 1966. Vertebrata. In: Borello, A.V. ed. Paleontografía Bonaerense. Buenos Aires, Com. Inv. Cient. Prov. n.4, p.1-12.

1984. La sucesión de las edades-mamífero, de los climas y del diastrofismo sudamericano durante el Cenozoico: fenómenos concurrentes. An. Acad. nac. Cienc. exact. fís. nat. B. Aires, Buenos Aires, 36:15-37.

Pascual, R. \& Rivas, O. E. O. 1971. Evolución de las comunidades de los vertebrados del Terciario argentino. Los aspectos paleozoogeográficos y paleoclimáticos relacionados. Ameghiniana, Buenos Aires, 7(3-4):372-412.

1973. Las unidades estratigráficas del Terciario portadoras de mamíferos. Su distribución y relaciones con los acontecimientos diastróficos. In: Congreso Geológico Argentino, $5^{\circ}$, Carlos Paz, 1972, Actas... v.3, p.295-338.

Perí, S. 1993. Ceratophrys (Anura, Leptodactylidae) en el Holoceno de Laguna Los Tres Reyes, provincia de Buenos Aires, Argentina. Ameghiniana, Buenos Aires, 30(1):3-7.

Pfretzschner, H.-U. 2000. Pyrite formation in Pleistocene bones - a case of very early mineral formation during diagenesis. N. Jb. Geol. Paläont. Abh., Stuttgart, 217(1):143-160.

Räsänen, M. E.; LinnA, A. M. et al. 1995. Late Miocene tidal deposits in the Amazonian foreland basin. Science, New York, 269:386-390.

ReIG, O. A. 1958. Notas para una actualización del conocimiento de la Formación Chapadmalal. Acta geol. lilloana, Tucumán, 2:255-283.

Rendel, J. M. 1965. The effects of genetic change at different levels. In: International Congress of Zoology, $16^{\circ}$, Proceedings... New York, J. A. Moore ed. v.6, p.285-295.

Reumer, J. W. F. \& Thiébaud, C. H. 1987. Osteocyte lacunae size in the genus Xenopus (Pipidae). Amphibia-Reptilia, Leiden, 8(4):315-320.

Ringuelet, R. A. 1978. Dinamismo histórico de la fauna brasílica en la Argentina. Ameghiniana, Buenos Aires, 15(1-2):255-262.

Rovereto, C. 1914. Los estratos araucanos y sus fósiles. An. Mus. nac. Hist. nat. B. Aires, Buenos Aires, 25:1-247.

RusConi, C. 1932. La presencia de anfibios ("Ecaudata") y de aves fósiles en el piso ensenadense de Buenos Aires. An. Soc. cient. argent., Buenos Aires, 113:145-149.

Spring, J. 1997. Vertebrate evolution by interspecific hybridisation; are we polyploid? FEBS Lett., Amsterdam, 400:2-8.

Stebins, G. L. 1966. Chromosome variation and evolution. Science, New York, 152:1463-1469.

Tonni, E. P. 1974. Un nuevo cariámido (Aves, Gruiformes ) del Plioceno superior de la provincia de Buenos Aires. Ameghiniana, Buenos Aires, 11:366-372.

Tonni, E. P. \& Fidalgo, F. 1978. Consideraciones sobre los cambios climáticos durante el Pleistoceno tardío-reciente en la provincia de Buenos Aires. Aspectos ecológicos y zoogeográficos relacionados. Ameghiniana, Buenos Aires, 15(1-2):235-253.

Uzzel, T.; Berger, L. \& GÜnther, R. 1975. Diploid and triploid progeny from a diploid female of Rana esculenta (Amphibia Salientia). Proc. Acad. nat. Sci. Philad., Philadelphia, 127:81-91.

Vergnaud-Grazzini, C. 1968. Amphibiens pléistocènes de Bolivie. Bull. Soc. géol. Fr., Paris, 10(7):688-695.

Vialli, M. 1971. Morfometria delle lacune ossee in Anfibi poliploidi. Boll. Zool., Napoli, 38:566-568.

Vialli, M. \& SACCHI-VIALLI, G. 1969. Morfometria delle lacune ossee di vertebrati attuali e fossili alla luce delle conoscenze di biologi cellulare. Instituto Lombardo (Rend. Sc.), Milán, (B)103:234-254.

Wendel, J. F. 2000. Genome evolution in polyploids. Plant. Mol. Biol., The Hague, 42(1):225-249.

Recebido em 17.09.2001; aceito em 06.06.2002 